\title{
Congestion occurrence and evaluation in acute heart failure scenario: author's replay to the letter
}

\author{
Alberto Palazzuoli ${ }^{1} \cdot$ Isabella Evengelista ${ }^{1}$ \\ Published online: 7 May 2020 \\ (C) Springer Science+Business Media, LLC, part of Springer Nature 2020
}

We would like to thank the author for his/her comments and for the suggestion to add new emerging biomarkers (sCD146) among the laboratory tools for congestion detection. We believe that although the cited biomarkers have potential insights for congestion recognition, currently few data support the introduction of sCD146 among the traditional laboratory algorithms. Indeed, most data came from single-center studies and only one research group (Arrigo et al.) has deeply investigated CD146 in heart failure (HF). These two items show only slight evidence for being included as current biomarkers in the much more common and recognized laboratory list [1]. We would like to point out that many emerging biomarkers in the past provided exciting results in preliminary phases that unfortunately after multicenter studies and larger scale population inclusion, did not confirm sufficient reproducibility and significant diagnostic accuracy [2].

Notably, each new biomarker to be tested in HF may reflect some chemical and clinical features: the most important chemical characteristics are feasibility, international standardization, accurate and reproducible analytical sensitivity, well recognized reference range, and possibly a good cost-effectiveness ratio. Additionally, practical application in clinical activity must be maintained: each biomarker may yield some additional information to clinicians compared with the traditional scenario, it should reflect an exact pathophysiological mechanism, it could be useful for stratifying and screening patients, and it should help in monitoring disease progression and therapy response [3]. A large number of biomarkers with a plausible biological application have been recently tested, most of them are related to inflammatory and neurohormonal dysfunctions

Alberto Palazzuoli

palazzuoli2@unisi.it

1 Department of Internal Medicine, Cardiovascular Diseases Unit, S. Maria alle Scotte Hospital Siena, University of Siena, Viale Bracci, 53100 Siena, Italy occurring in HF, but their real usefulness, diagnostic accuracy, and prognostic value are still to be proven [4]. Regarding the second comment on congestion management with loop diuretics, we agree that it could become a surrogate end point and it should be considered an early objective over the acute HF hospitalization. Specifically, the loop diuretic amount may be administered by looking at diuretic response and all the other clinical and diagnostic variables cited in the review [5].

Overall, we think that congestion is the most important factor in HF worsening and recurrence. Every clinical and diagnostic parameter that is useful to better evaluate and monitor this condition is welcome and encouraged.

\section{References}

1. Van Aelst LNL, Arrigo M, Placido R, Akiyama E, Girerd N et al (2018) Acutely decompensated heart failure with preserved and reduced ejection fraction present with comparable haemodynamic congestion. Eur J Heart Fail 20:738-747

2. Januzzi JL, Felker M (2013) Surfing the biomarker tsunami at JACC heart failure. JACC Heart Fail 1:213-215

3. Morrow DA, de Lemos JL (2007) Benchmarks for the assessment of novel cardiovascular biomarkers. Circulation. 115:949-952

4. de Boer RA, Daniels LB, Maisel A, Januzzi JL (2015) State of the art: newer biomarkers in heart failure. Eur J Heart Fail 17:559-569

5. Palazzuoli A, Ruocco G, Ronco C, McCullough PA (2015) Loop diuretics in acute heart failure: beyond the decongestive relief for the kidney. Crit Care 19:296. https://doi.org/10.1186/ s13054-015-1017-3

Publisher's note Springer Nature remains neutral with regard to jurisdictional claims in published maps and institutional affiliations. 